\title{
Cancer immunotherapy: unique perspectives for endocrine-related cancers
}

\author{
Joanne Y Y Ngeow ${ }^{1,2,3,4}$ and Laura S Ward ${ }^{5}$ \\ ${ }^{1}$ Cancer Genetics Service, Division of Medical Oncology, National Cancer Centre, Singapore, Singapore \\ ${ }^{2}$ Lee Kong Chian School of Medicine, Nanyang Technological University, Singapore, Singapore \\ ${ }^{3}$ Oncology Academic Clinical Program, SingHealth Duke NUS Medical School, Singapore, Singapore \\ 4 Institute of Cellular and Molecular Biology, Agency for Science, Technology and Research, Singapore, Singapore \\ 5Laboratory of Cancer Molecular Genetics, Faculty of Medical Sciences, University of Campinas (FCM-Unicamp), Rua \\ Tessalia Vieira de Camargo 126, Barao Geraldo, Campinas, Sao Paulo, Brazil
}

Correspondence should be addressed to J Ngeow or L S Ward Email

joanne.ngeow.y.y@ singhealth.com.sg or ward@ fcm.unicamp.br
The landscape of therapeutic options for patients with cancer has changed dramatically in the last decade. Advances in understanding the role of driver mutations in mediating tumor growth and progression, coupled with the development of molecular inhibitors for defined mutations, have given rise to precision oncology. Parallel to this development, significant progress in tumor immunotherapy has resulted in several new strategies for cancer therapy. Effective tumor immunotherapy strategies are designed to promote the cancer-immunity cycle and block the immune-suppressive effects of established tumors. Approaches have included checkpoint inhibitors that prevent T-cell unresponsiveness, toll-like receptor agonists, oncolytic viruses that induce necrotic tumor cell death and trigger systemic antitumor immunity and adoptive transfer of native and genetically engineered $\mathrm{T}$ cells that are designed to recognize specific tumor antigens through the T-cell receptor. The practicing oncologists and scientists will need to understand these developments intimately if they wish to take advantage of these therapeutic options.

In this special issue of Endocrine-Related Cancer, we intentionally highlight some of the unique perspectives related to the use of cancer immunotherapy in endocrinerelated cancers. Lucas Leite Cunha and coworkers review the main concepts regarding immune checkpoint mechanisms and how cancer cells disrupt these processes to undergo immune escape (Cunha et al. 2017). The authors update the use of established as well as novel immune checkpoint inhibitors across the various endocrine-related cancers including less common tumor subtypes such as neuroendocrine cancers and adrenocortical cancers. It is evident from their review that across the different tumor subtypes, there is tremendous variability in efficacy in using checkpoint inhibitors. This is in part because we are as yet unable to predict and define the tumor-specific (and context-specific) immunogenic peptides involved. It is now well established that the mutational load present in cancer cells correlate closely with T-cell infiltration of tumors. Stephan Gasser and coworkers review how the inherent immune signatures of individual tumor subtypes influence treatment outcomes and the current strategies in progress to try to identify peptides suitable for vaccine and chimeric antigen receptor (CAR) T-cell therapy (Gasser et al. 2017). Our inability to define the multiplicity of antigens that must be targeted to achieve significant clinical responses is discussed; progress in this area may prove critical as many endocrine-related cancers have a lower tumor mutation burden compared to the more immunogenic melanoma and lung cancers.

Endocrine-related cancers are also unique as many of them are hormone dependent or secrete hormones. This poses unique challenges in the use of immunotherapy as we do not as yet fully understand the implications of how 
the immune system responds to hormonal variations. We believe this is an important area that has been overlooked and merits special mention. We therefore dive deeper into these issues in the two reviews by Gamat \& McNeel and Mould and coworkers (Gamat \& McNeel 2017, Mould et al. 2017). In prostate cancer, androgen deprivation is a key strategy in the management of metastatic prostate cancer. Androgens and androgen deprivation have profound effects on the immune system. Melissa Gamat \& Douglas McNeel review this dynamic field and address the many unanswered challenges on how we best combine androgen deprivation with immunotherapy for the treatment of prostate cancer (Gamat \& McNeel 2017).

Similarly, the conventional management of thyroid cancer itself has significant impact on the immune system. Robert Mould and coworkers describe firstly the immunological cell subsets and the role they play in thyroid cancer tumorigenesis and how surgery, radioactive iodine and levels of thyroxine, 3,5,3'-triiodo-L-thyronine and thyroid-stimulating hormone modulate the immune system (Mould et al. 2017). The impact of thyroid-related hormones as well as other hormones on the immune system is an area in need of critical research attention. An additional area that merits increased research attention is that of immunerelated adverse effects. Clinical options for managing immune-related complications remain limited. They are currently treated with steroids and immunosuppressants, just as classical autoimmune and inflammatory diseases are, but with far less success. Priscilla Cukier and coworkers review the important topic of endocrine side effects of cancer immunotherapy and the treatment recommendations, should they occur (Cukier et al. 2017). Endocrine-related side effects range from hypopituitarism caused by hypophysitis, to thyroid and adrenal dysfunction, type 1 diabetes and rarely, hypoparathyroidism.

It is clear from this special issue that more research into the mechanism of immune reactions induced by immunotherapy is needed, but relatively little is being done. Although mouse models can be instrumental in dissecting mechanisms and testing treatments of human disease, they rarely recapitulate the adverse immune responses seen in patients. It is therefore up to treating clinicians to be vigilant in capturing the response as well as adverse events data to enable us to better integrate immunotherapy into mainstream oncology. Important areas of development include the need for better methods to monitor immune responses and better predictors of response and toxicity; the best timing of immunotherapy administration; duration of treatment and the combination between different strategies.

We hope the reader will enjoy exploring this issue and uncover the therapeutic promise these strategies pose for endocrine tumors.

Declaration of interest

The authors declare that there is no conflict of interest that could be perceived as prejudicing the impartiality of this editorial.

\section{Funding}

This work did not receive any specific grant from any funding agency in the public, commercial, or not-for-profit sector.

\section{References}

Cukier P, Santini FC, Scaranti M \& Hoff AO 2017 Endocrine side effects of cancer immunotherapy. Endocrine-Related Cancer 24 T331-T347. (doi:10.1530/ERC-17-0358)

Cunha LL, Marcello MA, Rocha-Santos V \& Ward LS 2017 Immunotherapy against endocrine malignancies: immune checkpoint inhibitors lead the way. Endocrine-Related Cancer 24 T261-T281. (doi:10.1530/ERC-17-0222)

Gamat M \& McNeel DG 2017 Androgen deprivation and immunotherapy for the treatment of prostate cancer. EndocrineRelated Cancer 24 T297-T310. (doi:10.1530/ERC-17-0145)

Gasser S, Lim LHK \& Cheung FSG 2017 The role of the tumour microenvironment in immunotherapy. Endocrine-Related Cancer 24 T283-T295. (doi:10.1530/ERC-17-0146)

Mould RC, van Vloten JP, AuYeung AWK, Karimi K \& Bridle BW 2017 Immune response in the thyroid cancer microenvironment: making immunotherapy a possible mission. Endocrine-Related Cancer $\mathbf{2 4}$ T311-T329. (doi:10.1530/ERC-17-0316)

Received in final form 19 September 2017 Accepted 20 September 2017
(C) 2017 Society for Endocrinology Printed in Great Britain
Published by Bioscientifica Ltd. 